\title{
A Comparative Study of LBE and DUGKS Methods for Nearly Incompressible Flows
}

\author{
Peng Wang ${ }^{1}$, Lianhua Zhu ${ }^{1}$, Zhaoli Guo ${ }^{1, *}$ and Kun $\mathrm{Xu}^{2}$ \\ 1 State Key Laboratory of Coal Combustion, Huazhong University of Science and \\ Technology, Wuhan, 430074, P.R. China. \\ ${ }^{2}$ Mathematics Department, Hong Kong University of Science and Technology, Clear \\ Water Bay, Kowloon, Hong Kong.
}

Received 24 June 2014; Accepted (in revised version) 17 October 2014

\begin{abstract}
The lattice Boltzmann equation (LBE) methods (both LBGK and MRT) and the discrete unified gas-kinetic scheme (DUGKS) are both derived from the Boltzmann equation, but with different consideration in their algorithm construction. With the same numerical discretization in the particle velocity space, the distinctive modeling of these methods in the update of gas distribution function may introduce differences in the computational results. In order to quantitatively evaluate the performance of these methods in terms of accuracy, stability, and efficiency, in this paper we test LBGK, MRT, and DUGKS in two-dimensional cavity flow and the flow over a square cylinder, respectively. The results for both cases are validated against benchmark solutions. The numerical comparison shows that, with sufficient mesh resolution, the LBE and DUGKS methods yield qualitatively similar results in both test cases. With identical mesh resolutions in both physical and particle velocity space, the LBE methods are more efficient than the DUGKS due to the additional particle collision modeling in DUGKS. But, the DUGKS is more robust and accurate than the LBE methods in most test conditions. Particularly, for the unsteady flow over a square cylinder at Reynolds number 300, with the same mesh resolution it is surprisingly observed that the DUGKS can capture the physical multi-frequency vortex shedding phenomena while the LBGK and MRT fail to get that. Furthermore, the DUGKS is a finite volume method and its computational efficiency can be much improved when a non-uniform mesh in the physical space is adopted. The comparison in this paper clearly demonstrates the progressive improvement of the lattice Boltzmann methods from LBGK, to MRT, up to the current DUGKS, along with the inclusion of more reliable physical process in their algorithm development. Besides presenting the Navier-Stokes solution, the DUGKS can capture the rarefied flow phenomena as well with the increasing of Knudsen number.
\end{abstract}

PACS: 44.05.+e, 47.11.-j, 47.56.+r

*Corresponding author. Email addresses: sklccwangpeng@hust.edu.cn (P. Wang), lhzhu@hust.edu.cn (L. Zhu), zlguo@hust.edu.cn (Z. Guo), makxu@ust.hk (K. Xu) 
Key words: Lattice Boltzmann equation, discrete unified gas-kinetic scheme, numerical performance.

\section{Introduction}

In recent years, the development of Boltzmann equation-based kinetic schemes has received particular attentions due to their distinctive modeling for flow simulations. The distinctive features in the kinetic methods include the following two aspects. Firstly, the Boltzmann equation provides a theoretical foundation for the hydrodynamic description from the underlying microscopic physics. Besides capturing the Navier-Stokes (NS) solutions, the kinetic methods can be used to study non-equilibrium flows in the transition regime. Secondly, the Boltzmann equation is a first-order integro-partial-differential equation with a linear advection term, while the Navier-Stokes equations are secondorder partial differential equations with a nonlinear advection term. The nonlinearity in the Boltzmann equation resides in its collision term, which is local. Therefore, the kinetic equation is more feasible to handle the discontinuities or unresolved flow regions. This feature may lead to some computational advantages for computational fluid dynamics (CFD) [1]. Due to the mesoscopic nature, kinetic methods are particularly appealing in modeling and simulating complex and non-equilibrium flows [2].

There have been a number of kinetic or mesoscopic methods, such as the lattice gas cellular automata (LGCA) [3], the lattice Boltzmann equation (LBE) [4], the gas-kinetic schemes (GKS) [5-7], and the smoothed particle hydrodynamics (SPH) [8]. Among these methods, the LBE and GKS methods are specifically designed for CFD. The kinetic nature of the LBE and GKS has led to many distinctive advantages that distinguish them from the classical CFD methods, and a variety of successful applications have been achieved [9-18]. Particularly, the lattice BGK (LBGK) $[19,20]$ model and multiple-relaxation-time (MRT) model, as two popular standard LBE methods, have been successfully applied and well-accepted for incompressible NS solutions [21,22]. With the improved collision model, the MRT has overcome the apparent defects in the LBGK $[22,23]$. Besides the standard LBE [24], which can be viewed as a special finite-difference scheme for the discrete velocity Boltzmann equation (DVBE) using a regular lattice associated with the discrete velocities, the LBE methods have many other variants. The extended LBE, which solve the DVBE using general finite-difference [25, 26], finite-volume (FV) [27-30], or finiteelement methods [31], can release the close coupling of the mesh and discrete velocities. As a result, arbitrary meshes can be employed in these generalized LBE methods. However, the decoupling in the extended LBE also destroys the nice features of the standard LBE. For example, many of the existing FV-LBE methods suffer from large numerical dissipation and poor numerical stability [28,29].

Recently, starting from the Boltzmann equation, a discrete unified gas-kinetic scheme (DUGKS) has been proposed for isothermal flow in all Knudsen regimes [7]. The DUGKS is a finite volume method, which combines the advantages of GKS in its flux modeling 
and LBE in its expanded Maxwellian distribution function and discrete conservative collision operator. Furthermore, the DUGKS has the asymptotic preserve (AP) property in capturing both rarefied and NS solutions in the corresponding flow regime [6]. The detailed construction of DUGKS can be found in [7], and it will be introduced briefly in the next section as well.

Although sharing a common kinetic origin, there are distinctive features in the LBE and DUGKS methods. The standard LBE methods are finite difference schemes, while the DUGKS is a finite volume one. Both LBE and DUGKS methods evolve in discrete phase space (physical and particle velocity space) and discrete time. In the LBE methods, the phase space and time step are coupled due to the particle motion from one node to another one within a time step. The DUGKS has no such a restriction and the time step is fully determined by the Courant-Friedrichs-Lewy (CFL) condition. In addition, the streaming modeling in LBE makes it difficult to be extended to non-uniform mesh, while the DUGKS has no difference to use uniform or non-uniform mesh, even unstructured mesh. More importantly, there are distinctive modeling difference in LBE and DUGKS in the particle evolution process. The LBE separates the particle streaming and collision process in its algorithm development. But, the particle transport and collision are fully coupled in DUGKS. This dynamic difference determines the solution deviation in their flow simulations.

The present work is motivated to provide a thorough comparative study of the LBE and DUGKS methods for nearly incompressible flows. The standard LBGK and MRT methods are chosen as the corresponding LBE models. In order to present a fair comparison, we mostly choose the same phase space discretization, even though the DUGKS is not limited to such a mesh arrangement. Two test cases in the incompressible limit, i.e., the two-dimensional cavity flow and the laminar flow past a square cylinder, will be used for comparison.

The remaining part of this paper is organized as follows. We first make a brief introduction of the LBGK, MRT and DUGKS methods in Section 2. The detailed comparisons of these three methods in terms of accuracy, stability, and efficiency, are given in Section 3. Section 4 is the conclusion.

\section{Numerical methods}

In this section, the LBGK, MRT and DUGKS will be introduced briefly first. More detailed descriptions can be found in the references. Among these three methods, both LBGK and DUGKS are based on the BGK model [32],

$$
\frac{\partial f}{\partial t}+\xi \cdot \nabla_{x} f=\Omega \equiv \frac{f^{e q}-f}{\tau}
$$

where $f=f(\boldsymbol{x}, \boldsymbol{\xi}, t)$ is the particle distribution function with particle velocity $\boldsymbol{\xi}$ at position $x$ and time $t$, and $f^{e q}$ is the Maxwellian equilibrium distribution function, 


$$
f^{e q}=\frac{\rho}{(2 \pi R T)^{D / 2}} \exp \left(-\frac{(\xi-u)^{2}}{2 R T}\right),
$$

where $R$ is the gas constant, $D$ is the spatial dimension, $\rho$ is the density, $u$ is the fluid velocity and $T$ is the temperature. For low Mach number flow, the Maxwellian distribution can be approximated by its Taylor expansion around zero particle velocity. As a result, the expanded discrete equilibrium distribution function becomes,

$$
f_{i}^{e q}=W_{i} \rho\left[1+\frac{\boldsymbol{\xi}_{i} \cdot \boldsymbol{u}}{R T}+\frac{\left(\boldsymbol{\xi}_{i} \cdot \boldsymbol{u}\right)^{2}}{2(R T)^{2}}-\frac{|\boldsymbol{u}|^{2}}{2 R T}\right]
$$

where $f_{i}^{e q}=\omega_{i} f^{e q}\left(\boldsymbol{\xi}_{i}\right), \omega_{i}=W_{i}\left(2 \pi R T_{1}\right)^{D / 2} \exp \left(\frac{\left|\xi_{i}\right|^{2}}{2 R T}\right)$, and $W_{i}$ is the weight coefficient corresponding to the particle velocity $\xi_{i}$. For isothermal and low speed flows, in each direction the three-point Gauss-Hermite quadrature is used to evaluate the moments, with the following discrete velocities and associated weights,

$$
\begin{aligned}
& \xi_{-1}=-\sqrt{3 R T}, \quad \xi_{0}=0, \quad \xi_{1}=\sqrt{3 R T} \\
& W_{0}=2 / 3, \quad W_{ \pm 1}=1 / 6 .
\end{aligned}
$$

In the simulations, the two-dimensional and nine velocity (D2Q9) model is employed in both DUGKS and LBE methods [19], which is generated using the tensor product method, and it can be written as

$$
\xi_{i}= \begin{cases}(0,0), & i=0 \\ (\cos [(i-1) \pi / 2], \sin [(i-1) \pi / 2]) c, & i=1-4, \\ (\cos [(2 i-9) \pi / 4], \sin [(2 i-9) \pi / 4]) \sqrt{2} c, & i=5-8\end{cases}
$$

where $c=\sqrt{3 R T}$, and the corresponding weight coefficients are $W_{0}=4 / 9, W_{1,2,3,4}=1 / 9$ and $W_{5,6,7,8}=1 / 36$.

Then, the discrete distribution function $f_{i}(\boldsymbol{x}, t)=\omega_{i} f\left(\boldsymbol{x}, \boldsymbol{\xi}_{i}, t\right)$ satisfies the following equation

$$
\frac{\partial f_{i}}{\partial t}+\xi_{i} \cdot \nabla_{x} f_{i}=\Omega_{i} \equiv \frac{f_{i}^{e q}-f_{i}}{\tau}
$$

The fluid density and velocity can be obtained from the discrete distribution functions,

$$
\rho=\sum_{i} f_{i}, \quad \rho \boldsymbol{u}=\sum_{i} \xi_{i} f_{i}
$$

\subsection{The LBGK model}

By integrating Eq. (2.6) from $t$ to $t+\Delta t$ along the characteristic line and evaluating the collision effect by averaging the values at the beginning and end of the trajectory, the 
evolution equation for the discrete distribution functions $\tilde{f}_{i}$ of LBGK can be obtained,

$$
\tilde{f}_{i}\left(\boldsymbol{x}_{j}+\boldsymbol{\xi}_{i} \Delta t, t_{n}+\Delta t\right)-\tilde{f}_{i}\left(\boldsymbol{x}_{j}, t_{n}\right)=-\frac{1}{\tau_{v}}\left(\tilde{f}_{i}\left(\boldsymbol{x}_{j}, t\right)-f_{i}^{e q}\left(\boldsymbol{x}_{j}, t\right)\right),
$$

where $\tau_{v}=\tau / \Delta t+0.5$ is the dimensionless relaxation time, and

$$
\tilde{f}_{i}=f_{i}-\frac{\Delta t}{2} \Omega_{i} \text {. }
$$

In the standard lattice Boltzmann method, the space is discretized with a uniform cartesian grid (or lattice) $\mathcal{L} \equiv\left\{x_{j}\right\}$, the time step $\Delta t$ is chosen according to the grid spacing such that a particle at $x_{i} \in \mathcal{L}$ will move to next node $x_{i}+\xi_{i} \Delta t \in \mathcal{L}$. Based on the compatibility condition and the relationship between $f_{i}$ and $\tilde{f}_{i}$, the density $\rho$ and velocity $u$ can be computed by

$$
\rho=\sum_{i} \tilde{f}_{i}, \quad \rho \boldsymbol{u}=\sum_{i} \xi_{i} \tilde{f}_{i} .
$$

Through the Chapman-Enskog expansion, the Navier-Stokes equations can be recovered from the LBGK equation. The viscosity in the Navier-Stokes equations is related to the dimensionless relaxation time by

$$
v=c_{s}^{2}\left(\tau_{v}-\frac{1}{2}\right) \Delta t
$$

where $c_{s}$ is the speed of sound.

\subsection{The MRT model}

Different from the LBGK, the collision step in the MRT model is executed in the moment space $\mathbf{m}:=\left\{m_{k}, k=0,1, \cdots, 8\right\}$ instead of the velocity space $\mathbf{f}:=\left\{f_{k}, k=0,1, \cdots, 8\right\}$. The evolution equation of MRT model is

$$
\tilde{\mathbf{f}}\left(\boldsymbol{x}_{j}+\boldsymbol{\xi}_{i} \Delta t, t_{n}+\Delta t\right)-\tilde{\mathbf{f}}\left(\boldsymbol{x}_{j}, t_{n}\right)=-\mathbf{M}^{-1} \mathbf{S}\left[\mathbf{m}-\mathbf{m}^{e q}\right],
$$

where the matrix $\mathbf{M}$ is used to transform the distribution function $\tilde{\mathbf{f}}$ and its equilibria $\mathbf{f}^{e q}$ to their moments $\mathbf{m}$ and $\mathbf{m}^{e q}$,

$$
\mathbf{m}=\mathbf{M} \cdot \tilde{\mathbf{f}}, \quad \mathbf{m}^{e q}=\mathbf{M} \cdot \mathbf{f}^{e q} .
$$

For the D2Q9 model, $\mathbf{M}$ is given by [22]

$$
\mathbf{M}=\left(\begin{array}{rrrrrrrrr}
1 & 1 & 1 & 1 & 1 & 1 & 1 & 1 & 1 \\
-4 & -1 & -1 & -1 & -1 & 2 & 2 & 2 & 2 \\
4 & -2 & -2 & -2 & -2 & 1 & 1 & 1 & 1 \\
0 & 1 & 0 & -1 & 0 & 1 & -1 & -1 & 1 \\
0 & -2 & 0 & 2 & 0 & 1 & -1 & -1 & 1 \\
0 & 0 & 1 & 0 & -1 & 1 & 1 & -1 & -1 \\
0 & 0 & -2 & 0 & 2 & 1 & 1 & -1 & -1 \\
0 & 1 & -1 & 1 & -1 & 0 & 0 & 0 & 0 \\
0 & 0 & 0 & 0 & 0 & 1 & -1 & 1 & -1
\end{array}\right) .
$$


With such a transformation matrix and the equilibrium distribution function $\mathbf{f}^{e q}$, the equilibria in the moment space is

$$
\mathbf{m}^{e q}=\rho\left(1,-2+3 u^{2}, 1-3 u^{2}, u_{x},-u_{x}, u_{y},-u_{y}, u_{x}^{2}-u_{y}^{2}, u_{x} u_{y}\right) .
$$

The relaxation matrix $\mathbf{S}$ in the MRT is a diagonal matrix. Its diagonal elements are the relaxation rates of the moments, i.e., $\mathbf{S}=\operatorname{diag}\left(0, s_{e}, s_{\varepsilon}, 0, s_{q}, 0, s_{q}, s_{v}, s_{v}\right)$, and the kinematic viscosity and bulk viscosity are related to the relaxation rates $s_{v}$ and $s_{e}$, respectively:

$$
v=c_{s}^{2}\left(\frac{1}{s_{v}}-\frac{1}{2}\right) \Delta t, \quad \zeta=c_{s}^{2}\left(\frac{1}{s_{e}}-\frac{1}{2}\right) \Delta t
$$

where $c_{S}$ is the speed of sound.

\subsection{The DUGKS model}

Unlike the LBE methods, the DUGKS is a finite-volume scheme. The computational domain is divided into a set of control volumes. Then integrating Eq. (2.6) over a control volume $V_{j}$ centered at $x_{j}$ from $t_{n}$ to $t_{n+1}$ (the time step $\Delta t=t_{n+1}-t_{n}$ is assumed to be a constant in the present work), and using the midpoint rule for the integration of the flux term at the cell boundary and trapezoidal rule for the collision term inside each cell [7], the evolution equation of DUGKS is

$$
\tilde{f}_{i, j}^{n+1}=\tilde{f}_{i, j}^{+, n}-\frac{\Delta t}{\left|V_{j}\right|} F_{i}^{n+1 / 2}
$$

where

$$
F_{i}^{n+1 / 2}=\int_{\partial V_{j}}(\boldsymbol{\xi} \cdot \boldsymbol{n}) f_{i}\left(\boldsymbol{x}, t_{n+1 / 2}\right) d \boldsymbol{S}
$$

is the flux across the cell interface, and

$$
\tilde{f}_{i}=f_{i}-\frac{\Delta t}{2} \Omega_{i}, \quad \tilde{f}_{i}^{+}=f_{i}+\frac{\Delta t}{2} \Omega_{i}
$$

Based on the compatibility condition and the relationship between $f_{i}$ and $\tilde{f}_{i}$, the density $\rho$ and velocity $\boldsymbol{u}$ can be computed by

$$
\rho=\sum_{i} \tilde{f}_{i}, \quad \rho \boldsymbol{u}=\sum_{i} \xi_{i} \tilde{f}_{i} .
$$

The key ingredient in updating $\tilde{f}_{i}$ is to evaluate the interface flux $F_{i}^{n+1 / 2}$, which is solely determined by the gas distribution function $f_{i}\left(\boldsymbol{x}, t_{n+1 / 2}\right)$ there. In DUGKS, after integrating Eq. (2.6) along a particle path, the evaluation of the gas distribution function $f_{i}\left(\boldsymbol{x}, t_{n+1 / 2}\right)$ at the cell interface can be traced back to the interior of neighboring cells,

$$
\bar{f}_{i}\left(x_{b}, \xi, t_{n}+h\right)=\bar{f}_{i}^{+}\left(x_{b}, \xi, t_{n}\right)-h \xi \cdot \sigma_{b},
$$


where

$$
\bar{f}_{i}=f_{i}-\frac{h}{2} \Omega_{i}, \quad \bar{f}_{i}^{+}=f_{i}+\frac{h}{2} \Omega_{i}
$$

$\bar{f}_{i}^{+}\left(x_{b}, \xi, t_{n}\right)$ and the gradient $\sigma_{b}=\nabla \bar{f}_{i}^{+}\left(x_{b}, \xi_{j}, t_{n}\right), h=\Delta t / 2$ can be approximated by linear interpolation. For example, in the one dimensional case, the reconstructions become

$$
\begin{aligned}
& \sigma_{j+1 / 2}=\frac{\bar{f}_{i}^{+}\left(\boldsymbol{x}_{j+1}, \boldsymbol{\xi}_{,}, t_{n}\right)-\bar{f}_{i}^{+}\left(\boldsymbol{x}_{j}, \boldsymbol{\xi}, t_{n}\right)}{x_{j+1}-x_{j}}, \\
& \bar{f}_{i}^{+}\left(\boldsymbol{x}_{j+1 / 2}, \boldsymbol{\xi}, t_{n}\right)=\bar{f}_{i}^{+}\left(\boldsymbol{x}_{j}, \boldsymbol{\xi}, t_{n}\right)+\sigma_{j+1 / 2}\left(x_{j+1 / 2}-x_{j}\right) .
\end{aligned}
$$

Note that the particle collision effect is included in the above evaluation of the interface gas distribution function. This is the key for the success of the DUGKS. Owing to the un-splitting treatment of the particle collision and transport process in the reconstruction of the distribution function at cell interfaces, the DUGKS is a self-adaptive scheme for different flow regimes. It has been shown in Ref. [7] that the reconstructed distribution function approaches to the Chapman-Enskog one at the Navier-Stokes level in the continuum limit, and to the free-transport one in the free-molecular limit.

Based on the compatibility condition and the relationship between $f_{i}$ and $\bar{f}_{i}$, the density $\rho$ and velocity $\boldsymbol{u}$ at the cell interface can be obtained,

$$
\rho=\sum_{i} \bar{f}_{i}, \quad \rho \boldsymbol{u}=\sum_{i} \xi_{i} \bar{f}_{i}
$$

from which the equilibrium distribution function $f^{e q}\left(x_{b}, \xi, t^{n}+h\right)$ at the cell interface can be obtained. Therefore, based on Eq. (2.22) and the obtained equilibrium state, the real gas distribution function at the cell interface can be determined from $\bar{f}_{i}$,

$$
f_{i}\left(x_{b}, t_{n}+h\right)=\frac{2 \tau}{2 \tau+h} \bar{f}_{i}\left(x_{b}, t_{n}+h\right)+\frac{h}{2 \tau+h} f^{e q}\left(x_{b}, t_{n}+h\right),
$$

from which the interface numerical flux can be evaluated.

In computations, we only need to follow the evolution of $\tilde{f}_{i}$ in Eq. (2.17). The required variables for its evolution are determined by

$$
\begin{aligned}
& \bar{f}_{i}^{+}=\frac{2 \tau-h}{2 \tau+\Delta t} \tilde{f}_{i}+\frac{3 h}{2 \tau+\Delta t} f^{e q}, \\
& \tilde{f}_{i}^{+}=\frac{4}{3} \bar{f}_{i}^{+}-\frac{1}{3} \tilde{f}_{i} .
\end{aligned}
$$

\section{Numerical results}

In this section, both the cavity flow and the laminar flow past a square cylinder in $2 \mathrm{D}$ will be simulated using the three methods. The accuracy, stability, and efficiency will be quantitatively evaluated. The LBGK and MRT models introduced in the last section 
are chosen as the LBE methods, whose results are compared with that from DUGKS. Since we will test the isothermal flow, the temperature $T$ is a constant, with $c_{s}=\sqrt{R T}$ and $R T=1 / 3$ in the simulations. For the DUGKS, the relaxation time is determined by $\tau=\mu / p$, where $\mu$ is the dynamic viscosity coefficient and $p=\rho R T$ is the pressure. The time step is determined by the CFL condition, i.e., $\Delta t=\eta \Delta x_{\min } / C$, where $\eta$ is the CFL number, $\Delta x_{\min }$ is the minimum grid spacing, and $C$ is the maximal discrete velocity.

\subsection{Cavity flow}

The two-dimensional lid-driven cavity flow is a standard benchmark problem for validating numerical schemes. The two-dimensional square cavity is covered by a Cartesian mesh. The top wall moves along the $x$-direction with a constant velocity $u_{0}$, and the other three walls are stationary. The flow is characterized by the Reynolds number $\operatorname{Re}=L u_{0} / v$, where $L$ is cavity length and $v$ is the shear viscosity coefficient. In the computation, the boundary length of the square cavity is 1.0, and the driven velocity is 0.1 . In DUGKS, the CFL number is fixed at $\eta=0.95$. Uniform mesh is employed in most calculations. The relaxation rates in the MRT are $s_{e}=1.64, s_{\varepsilon}=1.54, s_{q}=1.9$ and $s_{v}=1.0 / \tau_{v}$ [22], which are chosen by considering the numerical stability and the separation between hydrodynamic and kinetic modes [23]. The non-slip boundary conditions for LBE and DUGKS methods are implemented by the half way bounce-back [23] and bounce-back rule [7]. The upper corners are singular points, which are assumed to be stationary in the simulation. The convergent criterion is given by

$$
\frac{\sum|\boldsymbol{u}(t)-\boldsymbol{u}(t-1000 \Delta t)|}{\sum|\boldsymbol{u}(t)|}<10^{-6}
$$

where $u$ is the velocity, and $\Delta t$ is the time step.

First, the accuracy of LBGK, MRT and DUGKS is evaluated by comparing their velocity profiles at different Reynolds numbers. Fig. 1 shows the velocity profiles calculated by the DUGKS along the central lines at various Reynolds numbers with different mesh resolutions. It is observed that the DUGKS can give accurate solutions at all Reynolds numbers by using $128 \times 128$ mesh points. However, a fine mesh with $256 \times 256$ points has to be used in LBGK and MRT in order to get a stable solution at high Reynolds numbers. The velocity profiles from these three methods at $\operatorname{Re}=1000,5000,7500,10000$ are shown in Fig. 2, along with the benchmark data [33]. With the above two mesh resolutions, the LBGK can only give the stable solution at $\operatorname{Re}=1000$, and its numerical stability is inferior in comparison with MRT and DUGKS. Once the LBE methods are stable, almost identical solutions are obtained by these three methods. It should be noted that for MRT the location of the primary vortex at $\operatorname{Re}=10000$ is sensitive to the relaxation rate $s_{\varepsilon}$. For example, the MRT code gives a stable primary vertex with $s_{\varepsilon}=1.54$, but there is no such a stable primary vertex as $s_{\varepsilon}=1.8$.

Second, the pure stability of the LBE and DUGKS methods is evaluated without considering the accuracy of the results. For a stable solution, we measure the minimum 


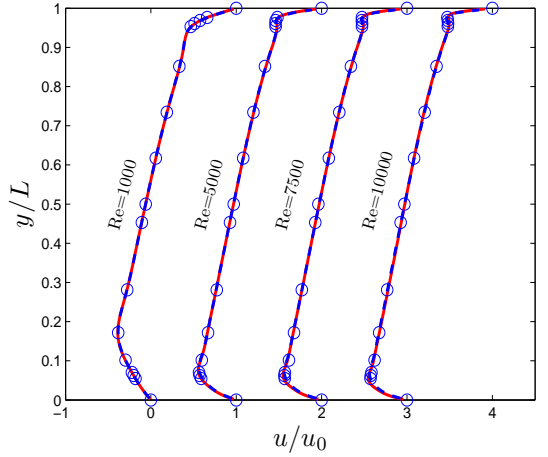

(a)

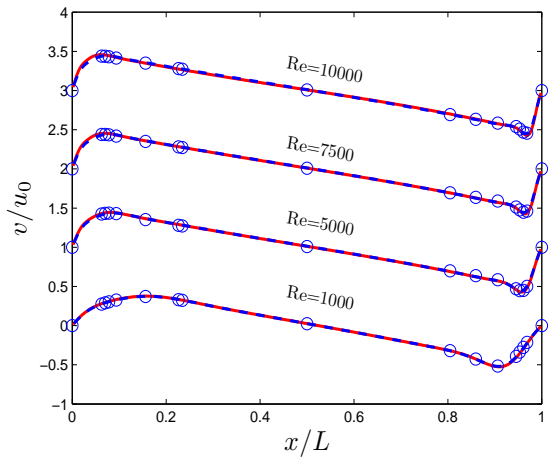

(b)

Figure 1: Velocity profiles for $(a) u$ and $(b) v$ calculated by DUGKS along a central lines passing through the geometric center of the cavity at various Reynolds numbers with difference meshes. The dash and solid lines are profiles for meshes $128 \times 128$ and $256 \times 256$, respectively, symbols are the benchmark results [33]. For convenient observation, lines for $\operatorname{Re}=5000,7500,10000$ are shifted along the axises.

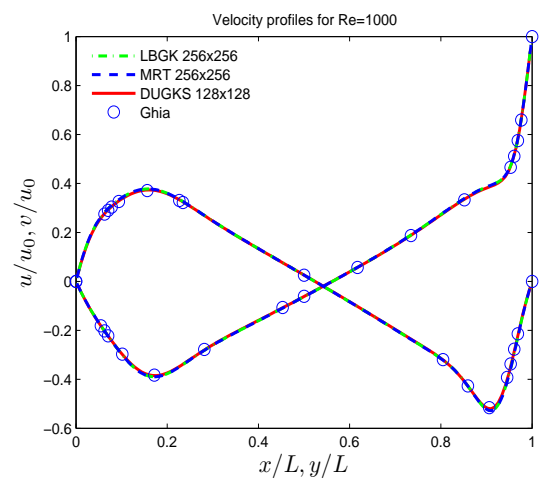

(a)

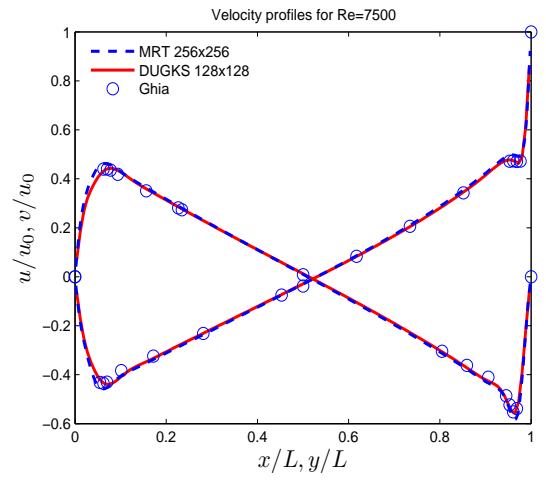

(c)

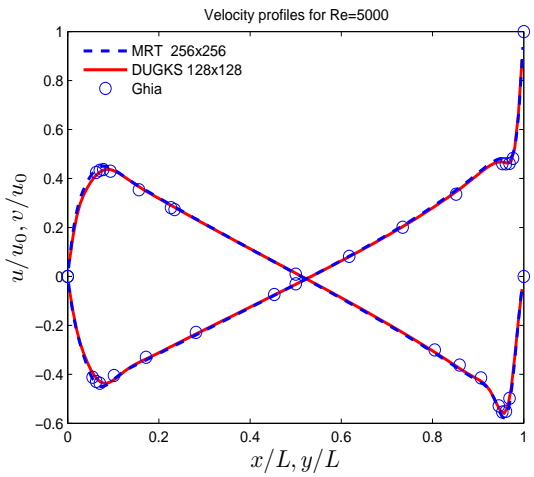

(b)

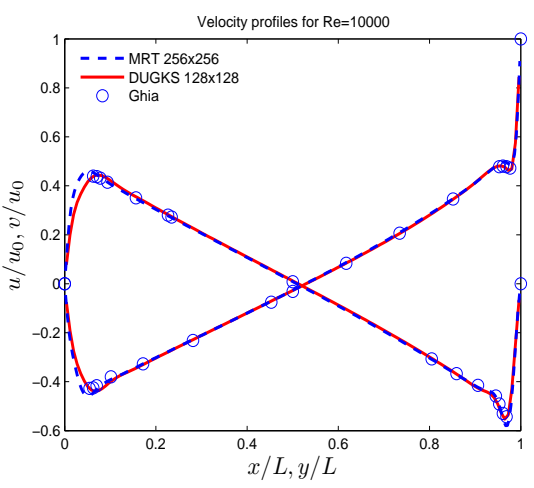

(d)

Figure 2: Velocity profiles calculated by LBKG, MRT, and DUGKS across the cavity center at (a) $\operatorname{Re}=1000$, (b) $\operatorname{Re}=5000,(c) \operatorname{Re}=7500,(d) \operatorname{Re}=10000$. The relaxation rates in MRT are $s_{e}=1.64, s_{\varepsilon}=1.54, s_{q}=1.9$ and $s_{v}=1.0 / \tau_{v}$. 


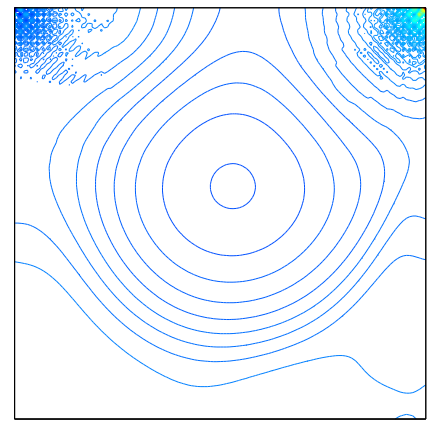

Figure 3: Pressure contour of the cavity flow calculated by $L B G K$ at $R e=1000$ on a $128 \times 128$ uniform mesh.

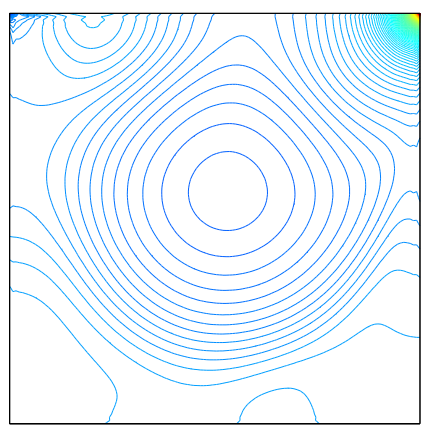

Figure 5: Pressure contour of the cavity flow calculated by DUGKS at $\mathrm{Re}=1000$ on a $128 \times 128$ uniform mesh

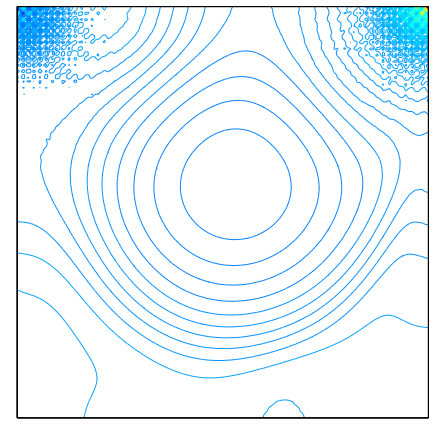

Figure 4: Pressure contour of the cavity flow calculated by MRT at $\mathrm{Re}=1000$ on a $128 \times 128$ uniform mesh with the relaxation rates $s_{e}=1.64, s_{\varepsilon}=1.54$, $s_{q}=1.9$ and $s_{v}=1.0 / \tau_{v}$

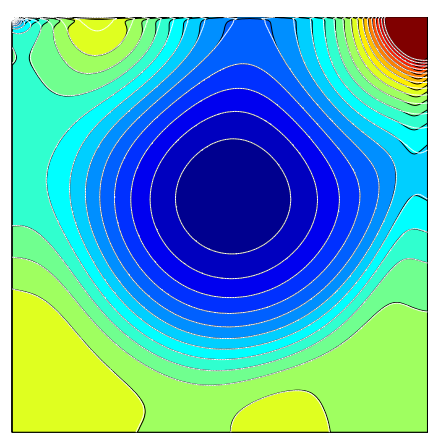

Figure 6: Pressure contour calculated by MRT with different $s_{e}$ at $\mathrm{Re}=1000$ on a $256 \times 256$ uniform mesh, where the black and white solid lines are from the calculations with $s_{e}=1.1$ and $s_{e}=1.9$, respectively. Other relaxation rates are given by $s_{\varepsilon}=1.0 / \tau_{v}, s_{q}=$ $8\left(2 \tau_{v}-1\right) /\left(8 \tau_{v}-1\right), s_{v}=1.0 / \tau_{v}$.

required mesh resolution at a fixed Reynolds number under steady state criterion of Eq. (3.1), and the maximum stable Reynolds number on a specific mesh resolution. Table 1 shows the minimum required mesh resolution at the given Reynolds numbers, where the DUGKS requires much less mesh points than the LBE methods in order to get a stable solution. For example, even at $\operatorname{Re}=10000$, the DUGKS can still use a $10 \times 10$ uniform mesh to reach a steady state solution. On the other hand, with a fixed mesh resolution, the DUGKS can reach a much higher Re than LBE methods. For instance, as shown in Table 2, on a uniform $128 \times 128$ mesh, the computations from the LBGK and MRT blow up at $\operatorname{Re}=1900$ and $\operatorname{Re}=8000$, respectively. However, the DUGKS works even at $\operatorname{Re}=100,000$. Clearly, in comparison with LBE methods, the DUGKS has super performance in stability. 
Table 1: The minimum required mesh resolution at different Reynolds numbers.

\begin{tabular}{||cccc||}
\hline Re & LBGK & MRT & DUGKS \\
\hline 1000 & $70 \times 70$ & $20 \times 20$ & $10 \times 10$ \\
5000 & $400 \times 400$ & $100 \times 100$ & $10 \times 10$ \\
7500 & $500 \times 500$ & $140 \times 140$ & $10 \times 10$ \\
\hline
\end{tabular}

Table 2: The maximum stable Re with different mesh resolutions.

\begin{tabular}{||cccc||}
\hline Mesh Size & LBGK & MRT & DUGKS \\
\hline $128 \times 128$ & 1900 & 8000 & 100000 \\
$256 \times 256$ & 3800 & 15000 & 350000 \\
\hline
\end{tabular}

Table 3: The CPU time costs when the numerical results are in good agreement with the benchmark data [33]. The symbol $\times$ means that the code blows up.

\begin{tabular}{||cccccc||}
\hline $\operatorname{Re}$ & LBGK $(128 \times 128)$ & LBGK $(256 \times 256)$ & MRT $(128 \times 128)$ & MRT $(256 \times 256)$ & DUGKS $(128 \times 128)$ \\
\hline 1000 & $110 s$ & $786 s$ & $297 s$ & $1574 s$ & $914 s$ \\
5000 & $\times$ & $\times$ & $\times$ & $8332 s$ & $4639 s$ \\
7500 & $\times$ & $\times$ & $\times$ & $12860 s$ & $7704 s$ \\
\hline
\end{tabular}

Third, in order to evaluate the computational efficiency, we measure the CPU times at various Reynolds numbers on different mesh resolutions. On the mesh of $128 \times 128$ points, the average CPU times for the update of one time step are $7.5 \times e^{-4}, 1.1 \times e^{-3}$ and $5.1 \times e^{-3}$ seconds for LBGK, MRT and DUGKS, respectively. Thus, the LBGK and MRT are about six and four times faster than DUGKS for each node update per time step. This is attributed to the four more equilibrium distribution evaluations in DUGKS in order to include collision effect into its flux computation. Table 3 shows the CPU times required to reach the steady-state solution (Eq. (3.1)) at the given Re and mesh resolutions. It is found that on the same mesh resolution, the LBGK and MRT are about eight and three times faster than the DUGKS to obtain the stationary solution. However, in order to obtain accurate solutions, the DUGKS can use a mesh with much less points in comparison with LBE methods. Therefore, in terms of obtaining accurate numerical solution the DUGKS and the LBE methods have almost the same computational efficiency in this case.

In addition, it is found that different pressure fields can be obtained from the LBGK, MRT, and DUGKS. The pressure contours predicted by the LBGK, DUGKS and MRT on the mesh of $128 \times 128$ points at $R e=1000$ are shown in Figs. 6-8. It is observed that un-physical pressure oscillations appear around the upper corners from the LBE computations, while a smooth pressure field can be obtained by DUGKS. In particular, for the MRT method, with the choice of another group of relaxation rates $s_{\varepsilon}=1.0 / \tau_{v}$, $s_{q}=8\left(2 \tau_{v}-1\right) /\left(8 \tau_{v}-1\right), s_{v}=1.0 / \tau_{v}$ and $s_{e}=1.0 / \tau_{v}$, which can be used to recover accurate non-slip bounce-back boundary condition [23], the un-physical oscillations disappear. However, the pressure field is sensitive to the value of $s_{e}$. The numerical stability of MRT 


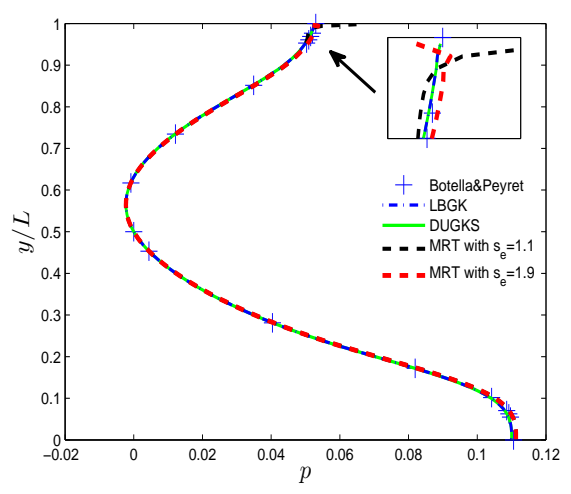

(a)

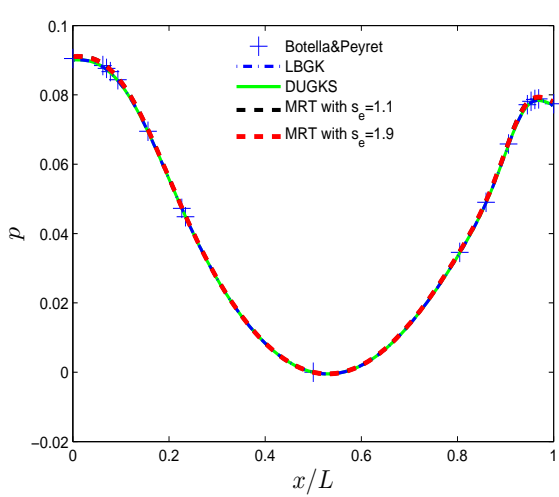

(b)

Figure 7: Pressure profiles along the central lines at $R e=1000$ on a $256 \times 256$ uniform mesh,(a) central vertical line, $(b)$ central horizontal line. The benchmark results are from [34]. The relaxation rates in MRT are given by $s_{\varepsilon}=1.0 / \tau_{v}, s_{q}=8\left(2 \tau_{v}-1\right) /\left(8 \tau_{v}-1\right), s_{v}=1.0 / \tau_{v}$.

deteriorates with the new relaxation values in comparison with the previous ones. For example, on a mesh of $256 \times 256$ points for $\mathrm{Re}=1000$ computation, the MRT gives two kinds of pressure fields near the top boundary, which are shown in Fig. 6, where the black and white solid lines represent the contours of pressure with $s_{e}=1.1$ and $s_{e}=1.9$, respectively. Fig. 7 shows the pressure profiles along the center of the cavity on the mesh of $256 \times 256$ points at $\operatorname{Re}=1000$, together with the benchmark data [34]. It is observed that the results from these three models are in good agreement with the reference data except the region near the top wall, where the prediction given by the MRT varies with the relaxation rates $s_{e}$.

\subsection{Laminar flow past a square cylinder in a channel}

This test case is the laminar flow past a square cylinder in a channel. The results from the LBGK, MRT, and DUGKS will be evaluated quantitatively. The square cylinder is symmetrically placed at the central line of a channel, which is shown in Fig. 8. The dimension of the cylinder is $D \times D$ within the channel with $L \times H$ in length $L$ and height $H$. The center of the cylinder is located at a distance of $L_{1}$ from the entrance. The flow configuration is defined by $L=50 D, H=8 D$, and $L_{1}=12.5 D$ in the simulations.

In a fully developed laminar channel flow, a parabolic velocity profile with a maximum velocity $U_{\max }$ is prescribed at the channel inlet. At the outlet, a convective boundary condition is applied [35],

$$
\partial_{t} \varphi+U_{\max } \partial_{x} \varphi=0
$$

where $\varphi$ is the flow variables or distribution function.

Depending on the Reynolds number $\operatorname{Re}=D U_{\max } / v$, different flow patterns can emerge. The critical Reynolds number, which is about $\operatorname{Re}=60$, classifies the flow into steady and 


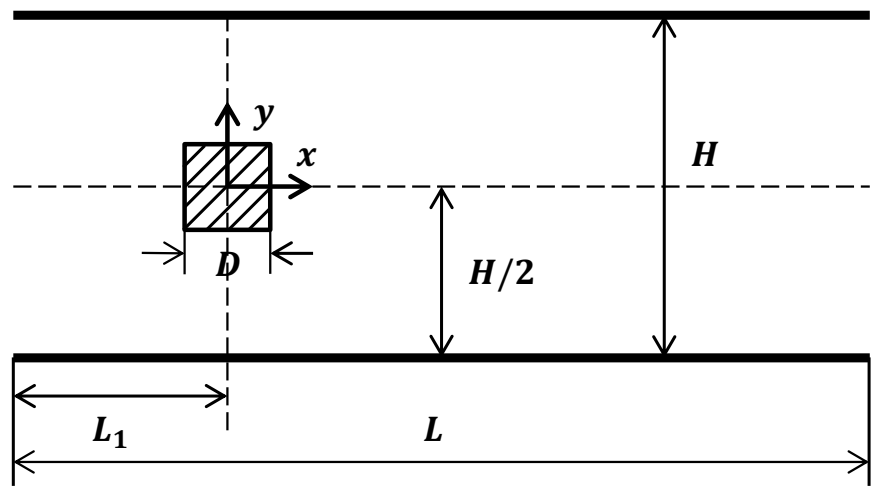

Figure 8: Schematic of the flow past a square cylinder in a 2D channel.

unsteady ones. When $\operatorname{Re}$ is below $\operatorname{Re}_{c}$, the flow is steady. Otherwise, the flow is unsteady and vortexes shed periodically in the wake region.

In the computations, two uniform meshes, i.e., a coarse one with $1500 \times 240$ mesh points and a fine one with $2000 \times 320$ mesh points, are used. In the coarse mesh case, there are 30 mesh points along each boundary of the square cylinder. The Mach number $M a=U_{\max } / c_{s}$ is set to be 0.1 for the incompressible limit. The relaxation rates in the MRT take the values of $s_{e}=1.1, s_{\varepsilon}=1.0, s_{q}=1.9$, and $s_{v}=1.0 / \tau_{v}[23,36]$. In the DUGKS, the CFL number is set to be $\eta=0.5$, unless otherwise stated. For the LBE and DUGKS methods, the half-way bounce back [36] and the bounce back rules [7] are respectively implemented for the non-slip boundary conditions at the top and bottom plates of the channel.

Besides the velocity field, the drag and lift coefficients will also be evaluated,

$$
C_{D}=\frac{F_{x}}{\frac{1}{2} \bar{\rho} U_{\max }^{2} D}, \quad C_{L}=\frac{F_{y}}{\frac{1}{2} \bar{\rho} U_{\max }^{2} D},
$$

where $\bar{\rho}$ is the mean fluid density, and $F_{x}$ and $F_{y}$ are the components of the hydrodynamic force on the square cylinder exerted by the fluid. The forces are computed by the integration method [36] in the DUGKS, and the momentum-exchange method [37] in the LBE methods.

The steady state solution is defined by

$$
\frac{\sqrt{\sum\|\boldsymbol{u}(t)-\boldsymbol{u}(t-1000 \Delta t)\|^{2}}}{\sqrt{\sum\|\boldsymbol{u}(t)\|^{2}}}<10^{-6},
$$

where $u$ is the fluid velocity. At low Reynolds number, the steady flow over a square cylinder is characterized by a pair of symmetric stationary recirculation eddies behind the cylinder. The length of the wake $L_{r}$ is a function of Re, which satisfies the following relationship [35],

$$
\frac{L_{r}}{D} \approx-0.065+0.0554 R e, \text { for } 5<\operatorname{Re}<60 .
$$




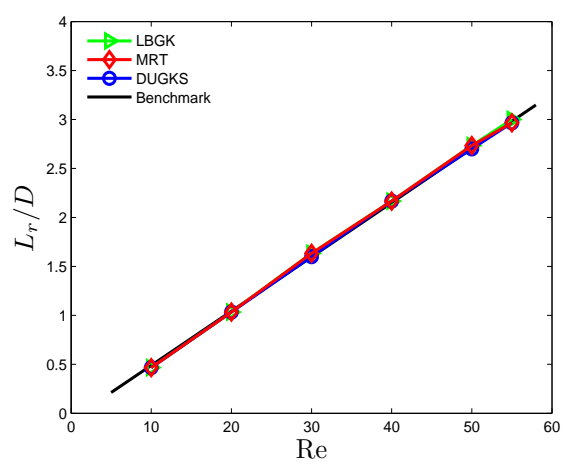

(a)

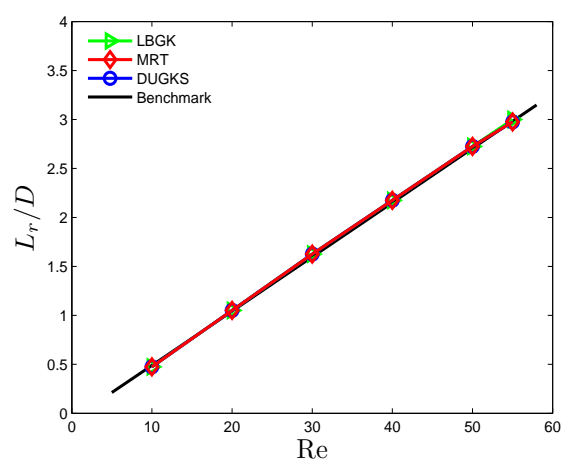

(b)

Figure 9: The Reynolds number dependence of the recirculation length $L_{r}$ in the steady state solution (a) results from $1500 \times 240$ mesh points, $(b)$ results from $2000 \times 320$ mesh points. The benchmark results are from [35].

In the current computation, $L_{r}$ is measured for the steady flow after satisfying the condition Eq. (3.4). Fig. 9 shows the measured relationships between the Reynolds number and $L_{r}$ on both coarse and fine meshes. The results obtained by LBGK, MRT and DUGKS are in excellent agreement with the linear function of Eq. (3.5) on both meshes. Fig. 10 and Fig. 11 present the velocity and pressure profiles at $\operatorname{Re}=30$ on the coarse and fine meshes. On both meshes, the velocity and pressure distributions given by these three methods agree well with each other.

For the steady state solution, the lift force is zero due to the flow symmetry, but the drag varies with Re. Fig. 12 shows the drag coefficients obtained by LBGK, MRT, and DUGKS on two meshes of $1500 \times 240$ points and $2000 \times 320$ points, along with the reference results obtained by a finite-volume method (FVM) [35]. The drag coefficient agrees well with the FVM data on both meshes. There is no difference in terms of the drag coefficient $C_{D}$ from the coarse and fine meshes for the steady state calculations, which indicates the mesh-size independent solutions at low Reynolds numbers.

Although the LBGK, MRT, and DUGKS present the same quantitative results at low Reynolds numbers, they have considerably different computational efficiency. On a uniform mesh of $1500 \times 240$ points, for the update of one time step the CPU costs of the LBGK, MRT and DUGKS are 0.023, 0.029, and 0.17 seconds, respectively. On a uniform mesh of $2000 \times 320$ points, the corresponding costs are $0.039,0.049$, and 0.29 seconds. Thus, the LBE methods are about five times faster than the DUGKS for the flow calculation per time step. In addition, the number of the time steps to achieve a steady-state solution are different in these three methods due to their different time accurate computations to the steady state. As shown in Tables 4 and 5, the LBE methods can reach to the steady-state, which is defined by Eq. (3.4), with one order of magnitude faster than DUGKS, even though all methods are supposed to present time accurate evolution solutions. The real physical time needed to get steady state is unknown here. The time costs of LBGK and DUGKS increase with Reynolds number on the same mesh resolution, while the MRT is 


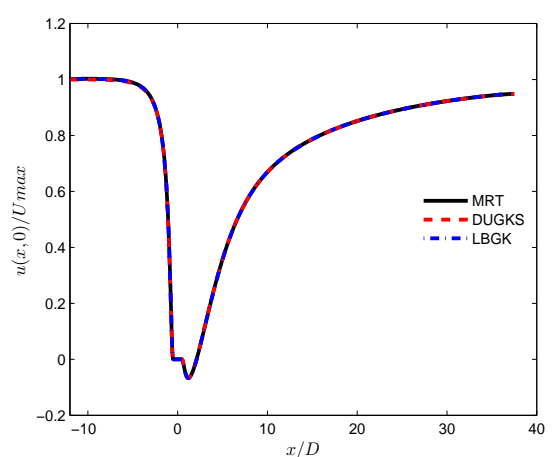

(a)

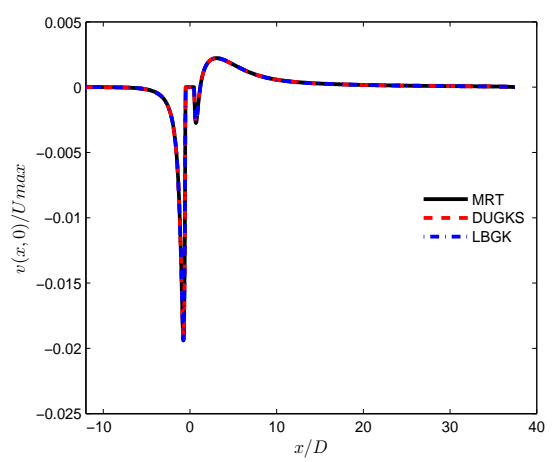

(c)

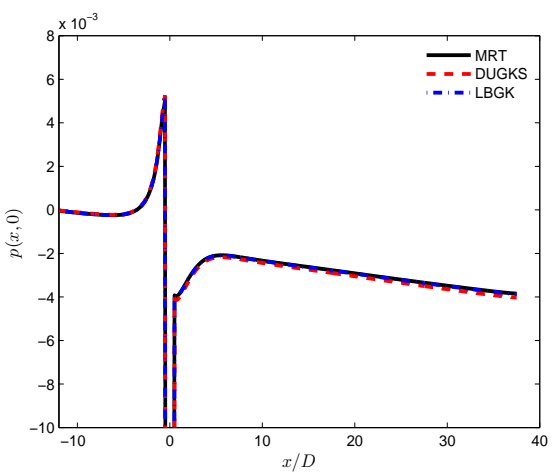

(e)

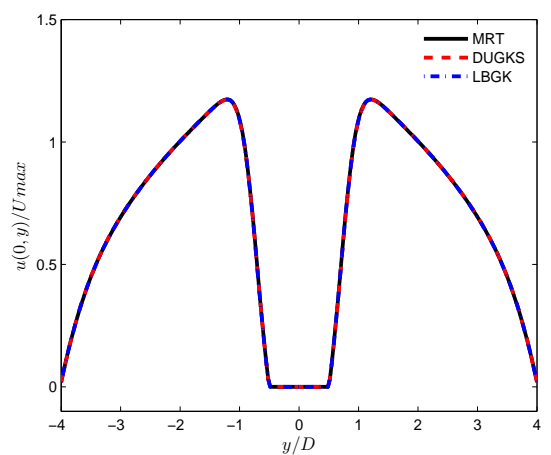

(b)

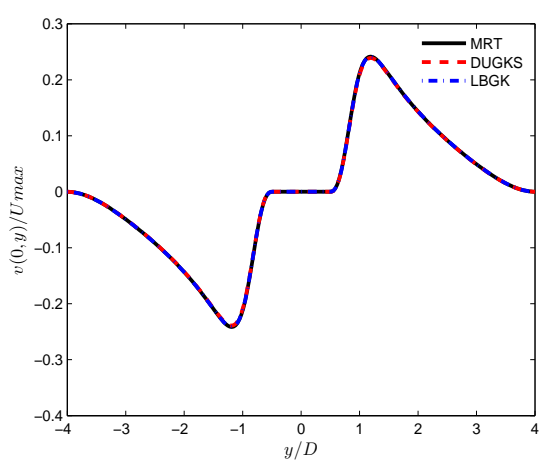

(d)

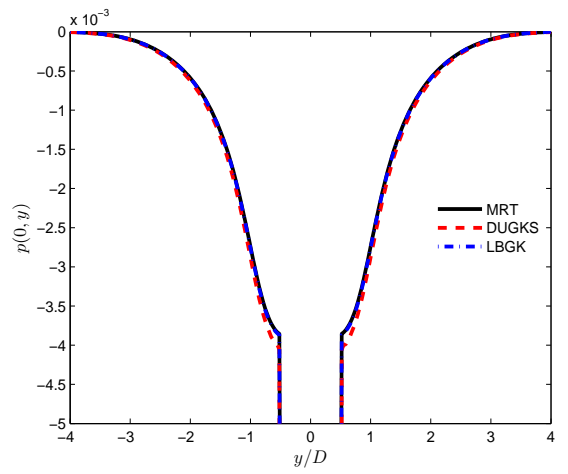

(f)

Figure 10: Velocity and pressure profiles at $\mathrm{Re}=30$ and $M a=0.1$ with a mesh of $1500 \times 240$ points. $(a) u(x, 0)$; (b)u(y,0); (c) v(x,0); (d) v(y,0); (e) $p(x, 0) ;(f) p(y, 0)$;

insensitive to Reynolds number due to its distinctive dissipation mechanism [23].

As the Reynolds number increases to the range of $60<R e \leq 300$, the recirculating wake behind the cylinder will become unstable. Two alternative shedding vortices will 


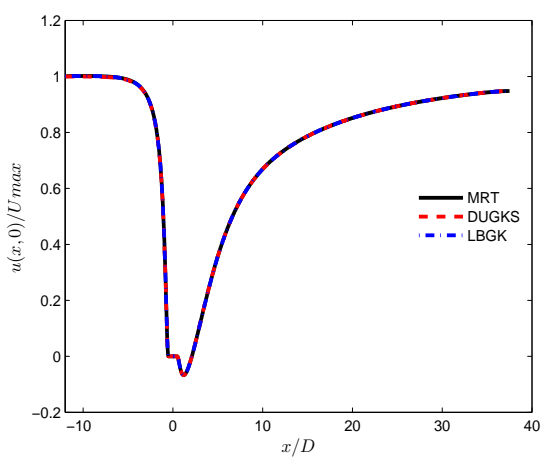

(a)

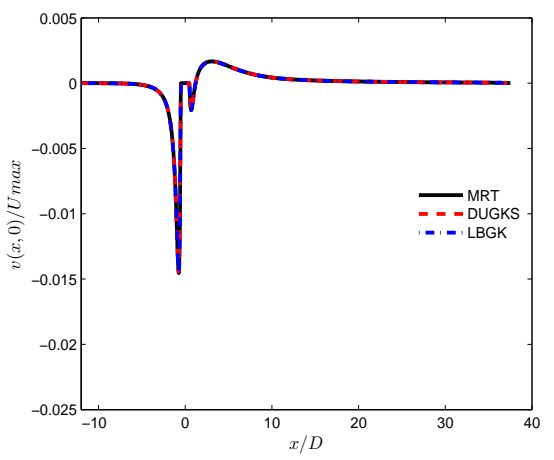

(c)

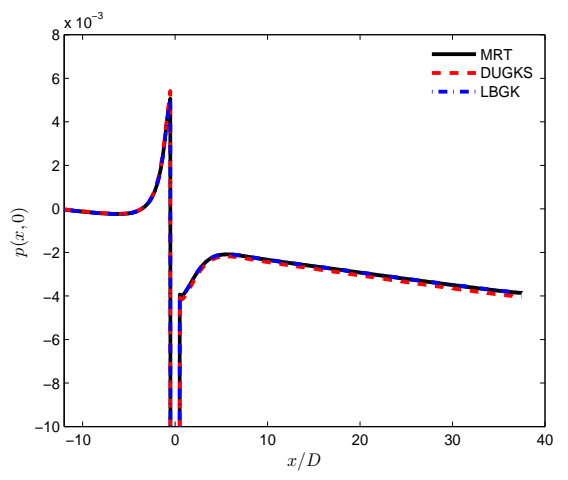

(e)

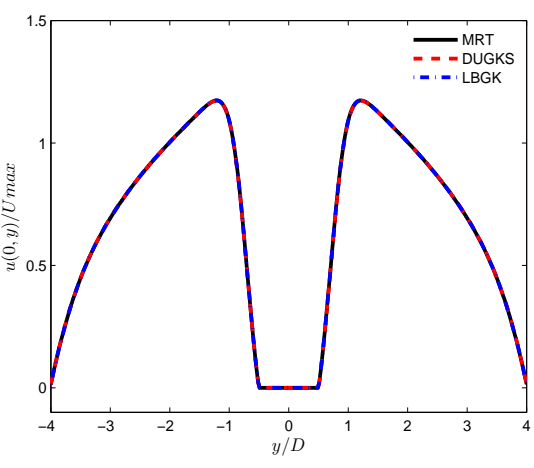

(b)

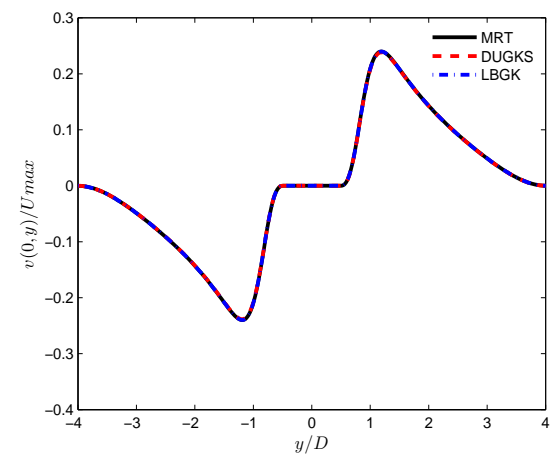

(d)

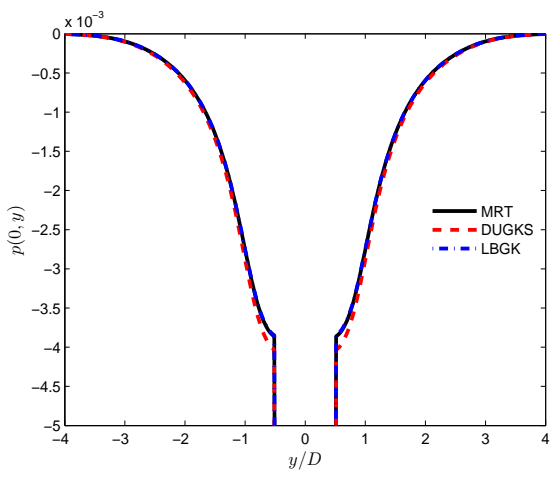

(f)

Figure 11: Velocity and pressure profiles at $\operatorname{Re}=30$ and $M a=0.1$ on the mesh of $2000 \times 320$ points. $(a) u(x, 0)$; $(b) u(y, 0) ;(c) v(x, 0)$; (d) $v(y, 0) ;(e) p(x, 0) ;(f) p(y, 0)$;

be formed in the rear part of the square cylinder, i.e., the so-called von Kármán vertex street appears. The characteristics of this unsteady flow can be measured by the mean and variation of the drag coefficient $\bar{C}_{D}$ and $\Delta C_{D}=C_{D}^{\max }-C_{D}^{\min }$, the variation of the lift 


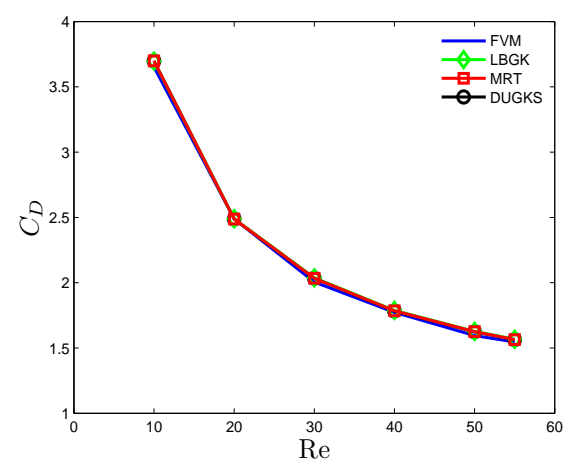

(a)

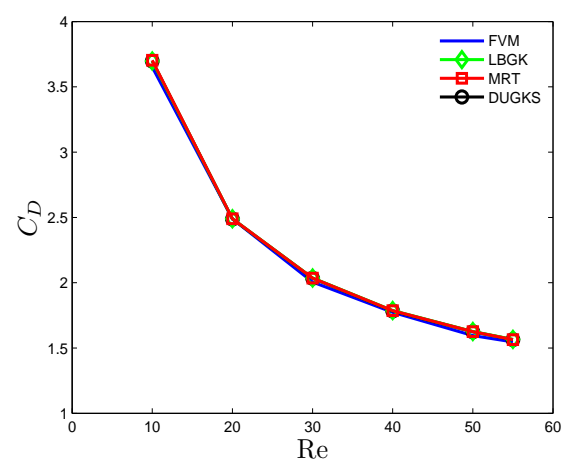

(b)

Figure 12: The Reynolds number dependence of the drag coefficient $C_{D}$ for steady state solutions. (a) results from $1500 \times 240$ mesh points, (b) results from $2000 \times 320$ mesh points. The results from another finite volume method (FVM) are also included [35].

Table 4: CPU times to attain the steady-state solution by using LBGK, MRT and DUGKS with a uniform mesh of $1500 \times 240$ points.

\begin{tabular}{||cccc||}
\hline Re & LBGK & MRT & DUGKS \\
\hline 10 & $1396 s$ & $1940 s$ & $20954 s$ \\
30 & $3160 s$ & $2381 s$ & $46065 s$ \\
50 & $4581 s$ & $3028 s$ & $72839 s$ \\
\hline
\end{tabular}

Table 5: CPU times to attain the steady-state solution by using LBGK, MRT and DUGKS with a mesh of $2000 \times 320$ points. A non-uniform mesh calculation with $280 \times 160$ points from DUGKS is also included.

\begin{tabular}{||ccccc||}
\hline Re & LBGK & MRT & DUGKS & DUGKS $(280 \times 160 \mathrm{NE})$ \\
\hline 10 & $3388 s$ & $4124 s$ & $51156 s$ & $3605 s$ \\
30 & $7708 s$ & $6235 s$ & $122304 s$ & $8547 s$ \\
50 & $10360 s$ & $7217 s$ & $189630 s$ & $12059 s$ \\
\hline
\end{tabular}

coefficient $\Delta C_{L}=C_{L}^{\max }-C_{L}^{\min }$, and the Strouhal number $S t=f_{s} D / U_{\max }$, where the superscripts max and min represent the maximum and minimum values, respectively, and $f_{s}$ is the vertex shedding frequency.

In order to validate these three methods, their results are compared with the reference data obtained by a FVM [35]. The FVM simulation uses a non-uniform mesh of $560 \times 340$, with 100 mesh points around each boundary of the square cylinder with a cell size of $0.01 D$. Fig. 13 presents the results of $\bar{C}_{D}, \Delta C_{D}, \Delta C_{L}$, and $S t$ from computations with a coarse uniform mesh of $1500 \times 240$. At the coarse mesh resolution, the overall results agree well with each other when $\operatorname{Re} \leq 200$, while the mean $C_{D}$ from the kinetic schemes is systematically higher than the reference solution. At the Reynolds number above 200, the LBGK, MRT, and DUGKS results deviate from the FVM data, especially the mean lift 


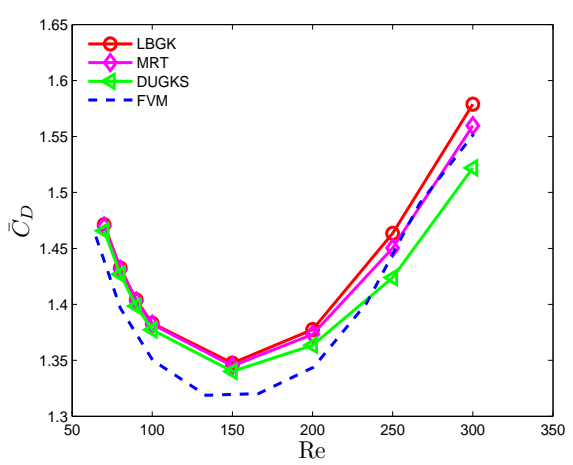

(a)

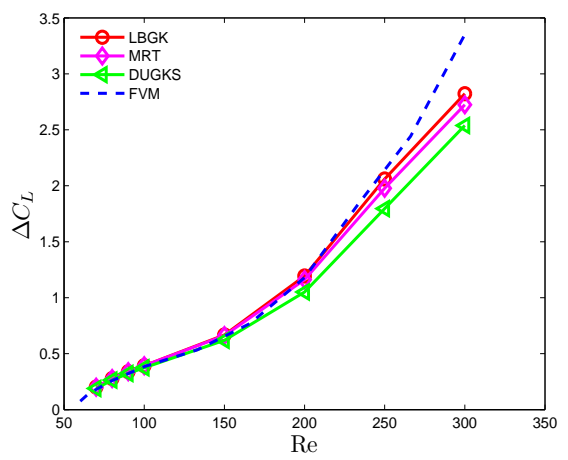

(c)

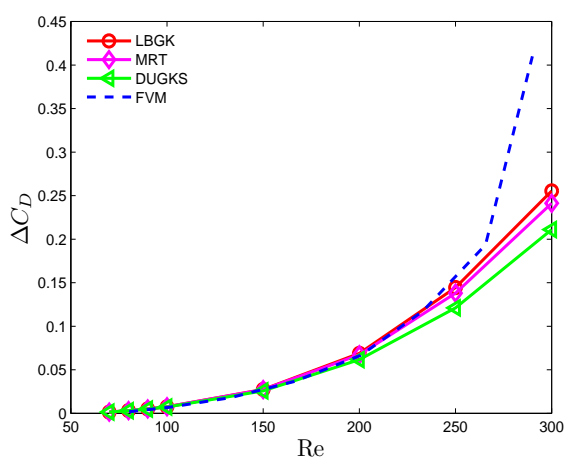

(b)

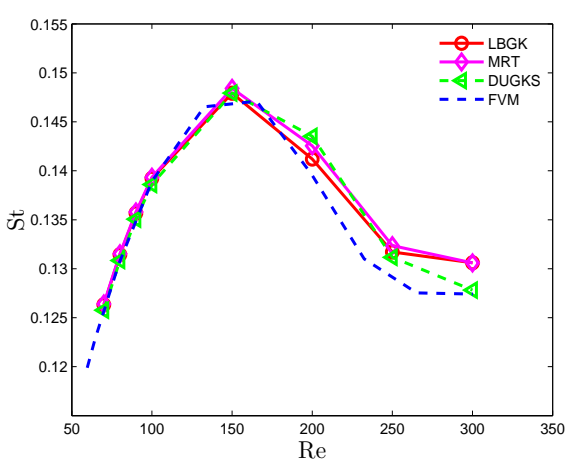

(d)

Figure 13: The Reynolds number dependence of $(a)$ the mean drag coefficient $\bar{C}_{D},(b)$ the variation of the drag coefficient $\Delta C_{D},(c)$ the variation of the lift coefficient $\Delta C_{L},(d)$ the Strouhal number $S t$, with a mesh of $1500 \times 240$ points. The results from another FVM are also included [35].

coefficients $\Delta C_{L}$ calculated by DUGKS at $\operatorname{Re}=300$, which can reach a maximum relative error of $24 \%$. This is attributed to the insufficient cell resolution with the coarse mesh in the region close to the cylinder with larger flow gradients.

With the increasing of mesh resolution to $2000 \times 320$, the results in Fig. 14 show a considerable improvement in comparison with the coarse mesh solutions. It is noted, however, that even with such a fine mesh resolution, it is still 2.5 times coarser around the square cylinder than the one used in the FVM computation. Quantitatively, the results given by LBGK, MRT, and DUGKS with the fine mesh agree well with the FVM data. The maximum relative error of $\Delta C_{L}$ calculated by DUGKS is down to $11 \%$. The maximum relative errors of $\bar{C}_{D}$ and $S t$ up to $\operatorname{Re}=300$, and $\Delta C_{D}$ up to $\operatorname{Re} \leq 250$, are less than $2 \%$. The discrepancy can be attributed to the compressibility effect which is on the order of $\mathcal{O}\left(\mathrm{Ma}^{2}\right)$. Thus, the results obtained by LBGK, MRT, and DUGKS are in excellent agreement with the FVM data up to $R e=250$.

It should be noted that the drag coefficient variation $\Delta C_{D}$ predicted by DUGKS in- 


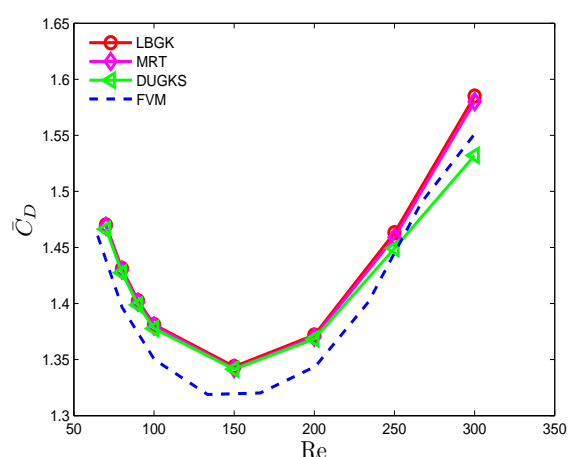

(a)

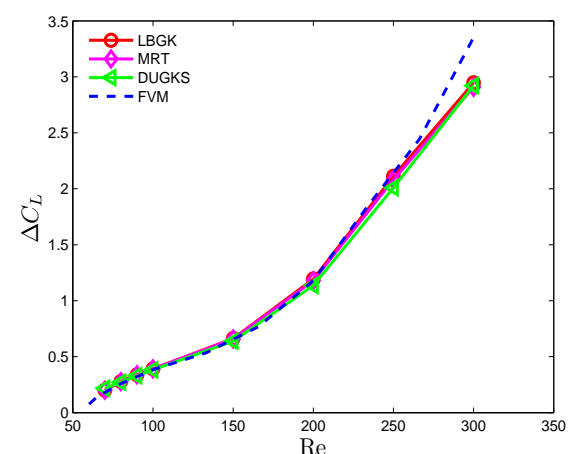

(c)

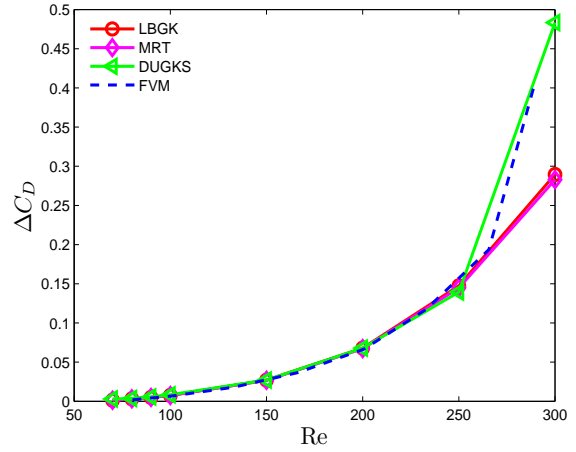

(b)

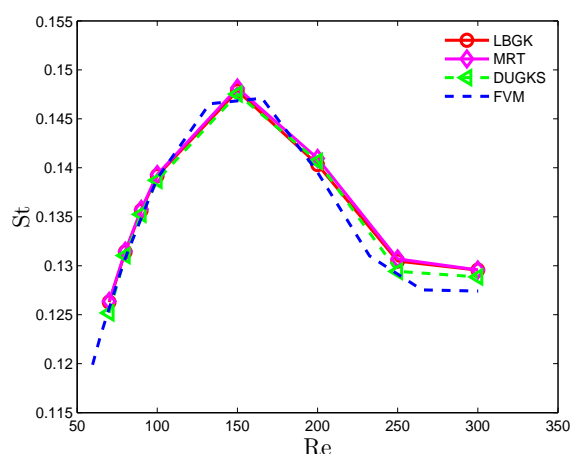

(d)

Figure 14: The Reynolds number dependent $(a)$ the mean drag coefficient $\bar{C}_{D} ;(b)$ the variation of the drag coefficient $\Delta C_{D} ;(c)$ the variation of the lift coefficient $\Delta C_{L} ;(d)$ the Strouhal number $S t$, from the calculations with $2000 \times 320$ mesh points. The reference results from a FVM is also included [35].

creases progressively in the high Reynolds number range, i.e., around $\operatorname{Re}=300$, which is consistent with the results obtained by FVM. But, the LBE methods don't capture such a phenomenon. In particular, at $\operatorname{Re}=300$, as shown in Fig. 15, there are different predictions of drag and lift forces from the LBE and DUGKS methods on the fine mesh calculations. The LBE methods presents a single frequency oscillations, while the DUGKS captures the complex multi-frequency periodic flows. The multiple frequency oscillating phenomenon obtained by DUGKS is also observed in the FVM [35] and finite element method (FEM) simulations [38]. The complex periodic oscillating flow should be a physical reality which identifies the flow transition at $\operatorname{Re}=300$. Therefore, even for the laminar flow the DUGKS has a better capability to capture physical phenomena than the LBE methods.

In addition, we investigate the stability of LBGK, MRT, and DUGKS. With a mesh resolution of $1500 \times 240$, LBGK and MRT codes blow up at $\operatorname{Re} \approx 1,000$ and $\operatorname{Re} \approx 5,000$, respectively. For the DUGKS with CFL number $\eta=0.5$, it can give stable solutions up to 


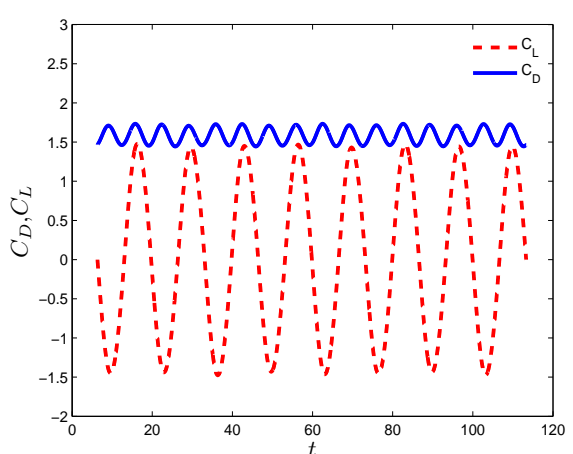

(a)

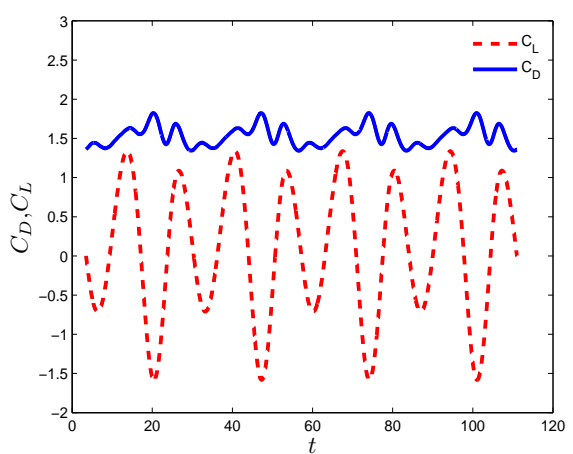

(c)

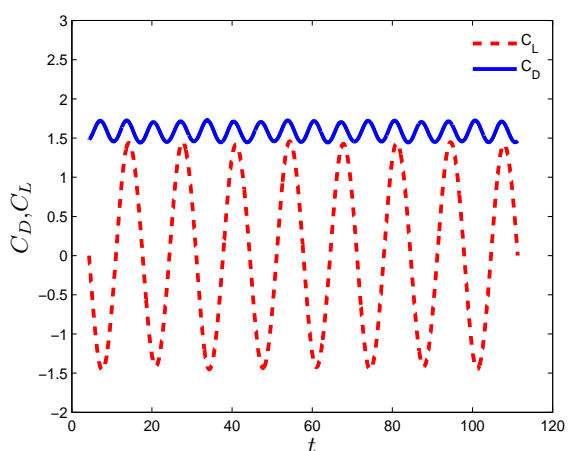

(b)

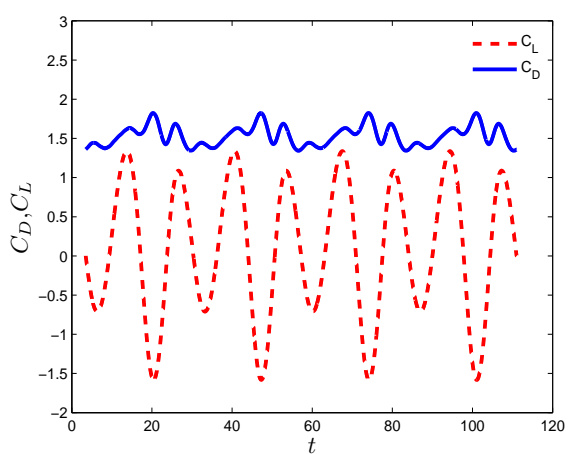

(d)

Figure 15: The time history of the drag coefficient $C_{D}$ and lift coefficient $C_{L}$ at $\operatorname{Re}=300$. (a) LBGK with a uniform mesh of $2000 \times 320$; (b) MRT with a uniform mesh of $2000 \times 320$; (c) DUGKS with a uniform mesh of $2000 \times 320 ;(d)$ DUGKS with a non-uniform mesh of $280 \times 160$.

$\operatorname{Re} \approx 30,000$. Same as the cavity flow computation, the DUGKS is more robust than the LBE methods for the flow past a square cylinder simulation as well.

Finally, we demonstrate the effectiveness of the DUGKS when using non-uniform mesh. The finite-volume nature of the DUGKS makes it easy to vary the mesh resolution according to the local accuracy requirement. Consequently, the overall total mesh points can be much reduced. For the current test, a non-uniform mesh is generated and its solution will be compared with the one from uniform mesh. The non-uniform mesh is defined by:

$$
x_{i}=L \frac{\exp (b i / N)-1}{\exp (b)-1}
$$

where $x_{i}$ is the location of the cell center, $N$ is the number of mesh points in front and at the rear parts of the square cylinder, $b$ is the stretching parameter, and $L$ is the length from the cylinder face to the boundary. In the following simulation, a non-uniform mesh of $280 \times 160$ points is generated, with 40 grids uniformly distributed around the surface 


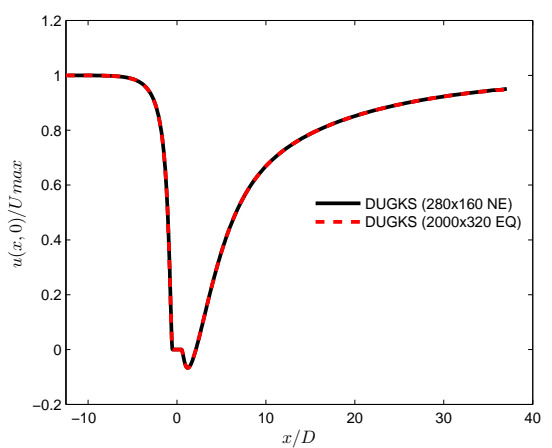

(a)

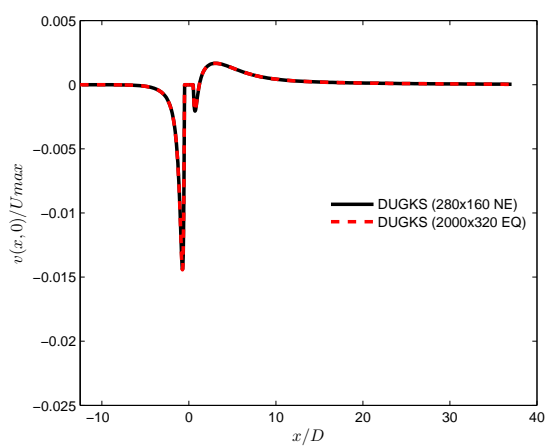

(c)

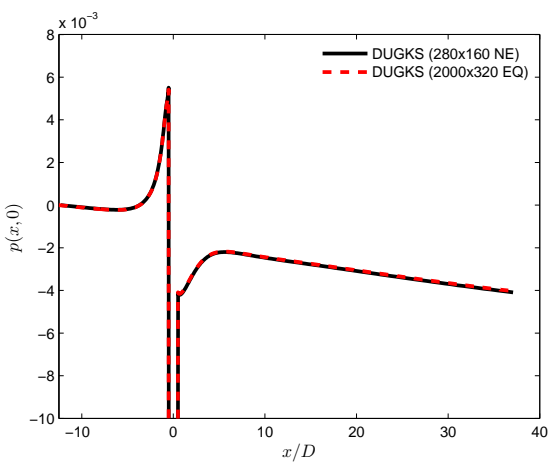

(e)

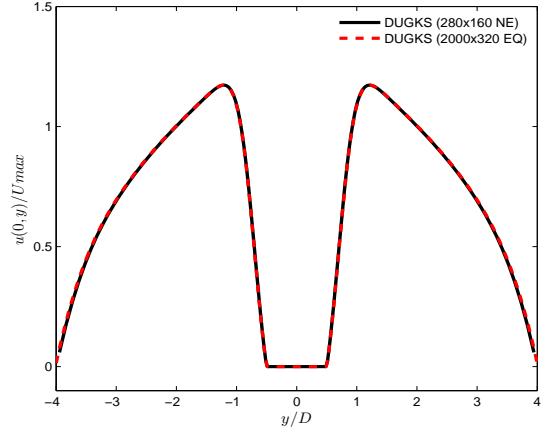

(b)

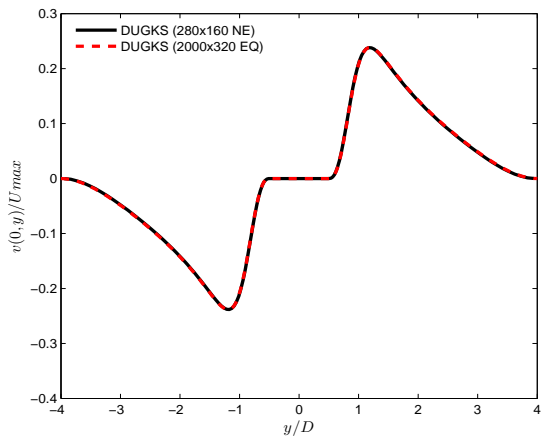

(d)

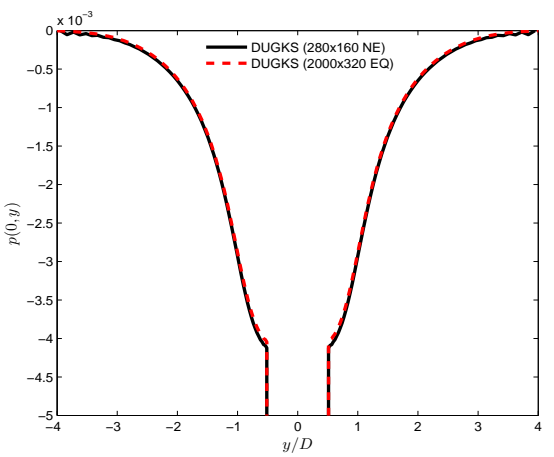

(f)

Figure 16: Velocity and pressure profiles from DUGKS at $\operatorname{Re}=30$ and $M a=0.1$ with a non-uniform mesh of $280 \times 160$ and a uniform mesh of $2000 \times 320$. (a) $u(x, 0) ;(b) u(y, 0) ;(c) v(x, 0) ;(d) v(y, 0) ;(e) p(x, 0) ;(f)$ $p(y, 0)$;

of the square, which has the same cell size on the surface of the cylinder as the uniform fine mesh calculation with $2000 \times 320$ mesh points. The expansion parameters $b$ takes $2.9,3.5,3.5,3.5$ on the left, right, top, and bottom of the square cylinder, respectively. 


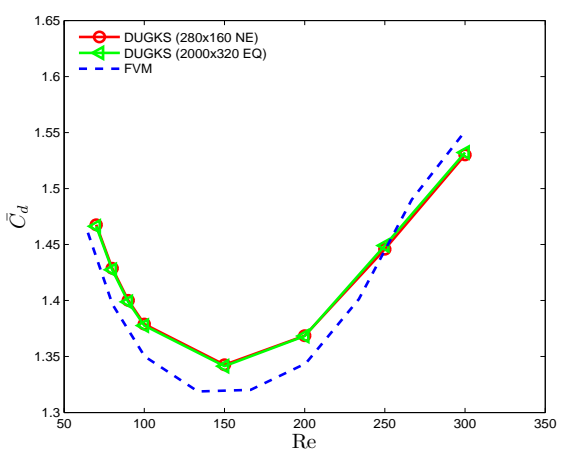

(a)

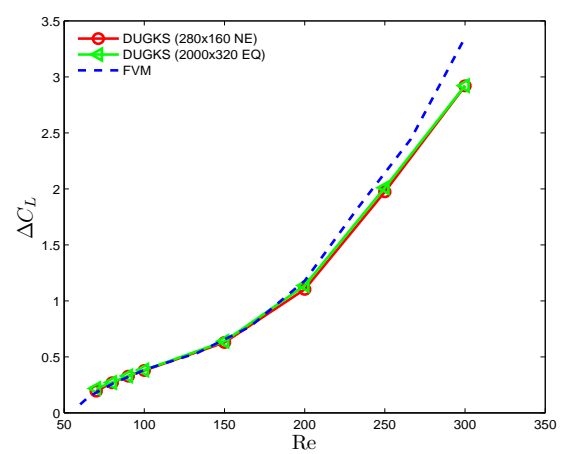

(c)

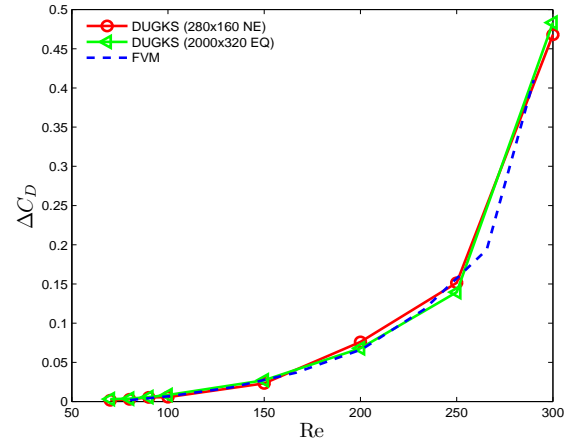

(b)

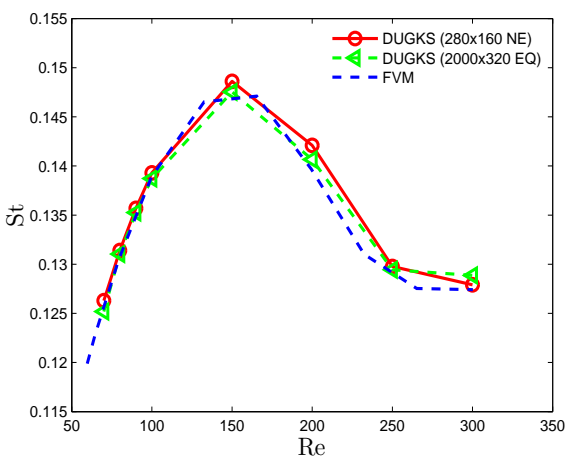

(d)

Figure 17: The Reynolds number dependent $(a)$ the mean drag coefficient $\bar{C}_{D} ;(b)$ the variation of the drag coefficient $\Delta C_{D} ;(c)$ the variation of the lift coefficient $\Delta C_{L} ;(d)$ the Strouhal number $S t$, with a non-uniform meshes of $280 \times 160$, and a uniform mesh of $2000 \times 320$. The reference results from a FVM is included [35].

Fig. 16 shows velocity and pressure profiles computed by DUGKS at $\operatorname{Re}=30$ with a non-uniform mesh, which have excellent agreement with the results with refined uniform mesh $2000 \times 320$. Fig. 17 displays that the DUGKS results of $\bar{C}_{D}, \Delta C_{D}, \Delta C_{L}$, and $S t$ at various Reynolds number from both the refined uniform and non-uniform meshes, and the reference solutions from the FVM simulation [35]. Almost identical accurate results can be obtained by DUGKS from two meshes. But, the computational efficiency is significantly different by using the uniform and non-uniform meshes. For example, on the $280 \times 160$ non-uniform mesh points, the CPU time cost is 0.0185 seconds per time step, which is about 14 times faster than that on a uniform mesh of $2000 \times 320$. As shown in Table 5, on a non-uniform mesh the DUGKS can reach to a steady state with 13 times less computational time than that on a fine uniform mesh. Therefore, the efficiency of DUGKS can be much improved by adopting a non-uniform mesh. The use of local time for the steady state calculation is another choice for DUGKS. 


\section{Conclusion}

In this paper, a quantitative comparison of the numerical performance from the LBE methods (LBGK and MRT) and DUGKS has been conducted. Both the cavity flow and the laminar flow passing through a square cylinder have been simulated in the nearly incompressible limit. The numerical accuracy, stability, and computational efficiency of these methods have been presented.

The current LBE methods and DUGKS use the same numerical discretization in the particle velocity space, such as the use of 9 particle velocities in the 2D computation. But, their discretization in the physical space is rather different. As a finite volume method, the DUGKS includes the particle collision in the transport process for the interface flux evaluation. As a result, the strict connection between the particle velocity and physical space discretization in the LBE methods can be totally released.

The LBE methods and DUGKS yield comparable results in all test cases when sufficient mesh resolution is provided. However, in the cavity flow computation, un-physical pressure oscillations occur from the LBE methods, while a smooth pressure field can be obtained by DUGKS. The un-physical pressure oscillations disappear when appropriate relaxation rates are used in MRT. But, they may give non-unique solutions with different choices of relaxation rates. In the flow passing through the square cylinder case, the DUGKS seems have large numerical dissipation in comparison with the LBE methods. This is mainly due to the dissipation introduced in DUGKS through the initial data reconstruction, which is absent in the LBE methods. However, at $\operatorname{Re}=300$, the multifrequency vortex shedding has been captured by DUGKS, which is consistent with other FVM [35] and FEM [38] results, and this phenomenon has been observed by DUGKS on a non-uniform coarse mesh as well. Unfortunately, both LBGK and MRT methods fail to capture the multiple frequency flow oscillation under the same mesh resolution.

Although LBE methods and DUGKS have similar accuracy in the simulations, they show considerable differences in terms of robustness. In the cavity flow simulation, the DUGKS needs a much lower mesh resolution to reach a steady-state solution than that used in the LBE methods. With the same mesh resolution, the DUGKS can simulate the flow at a much higher Reynolds number than the LBE methods. The same conclusion can be drawn for the flow over a square cylinder case as well.

In terms of efficiency, the DUGKS is four times slower than LBE methods in floating point operations (FLOPs) at each node per time step due to its additional physical modeling for the flux evaluation. However, as a finite volume scheme the DUGKS can use non-uniform mesh easily. As a result, the non-uniform mesh can be clustered in the region with large flow gradient. Therefore, the efficiently of DUGKS can be much improved. As expected, for the flow passing through the square cylinder case, the DUGKS code with a non-uniform mesh is about 13 times faster than that with a uniform mesh for the same accurate solution.

In conclusion, the DUGKS and LBE methods have similar accuracy for the flow simulations. But, the DUGKS is superior to the LBE methods in terms of numerical stability. 
With the same mesh resolution, the LBE methods are more efficient than the DUGKS. But, with the implementation of non-uniform mesh, the computational efficiency of the DUGKS can be greatly improved. The comparison presented in this paper clearly demonstrates the progressive improvement of the lattice Boltzmann methods from LBGK, to MRT, up to the current DUGKS.

\section{Acknowledgments}

P. Wang, L.H. Zhu and Z.L. Guo acknowledge the support by the National Natural Science Foundation of China (51125024) and the Fundamental Research Funds for the Central Universities (2014TS119). K. Xu was supported by Hong Kong research grant council (621011, 620813).

\section{References}

[1] B. van Leer, Computational fluid dynamics: science or toolbox, AIAA Report No. 2001-2520, 2001.

[2] M. Karttunen, I. Vattulainen, and A. Lukkarinen (Eds.), Novel Methods in Soft Matter Simulations, vol. 640 of Lecture Notes in Physics, Springer, Berlin, 2004.

[3] D.H. Rothman and S. Zaleski, Lattice-gas cellular automata: simple models of complex hydrodynamics, Cambridge University Press, 2004.

[4] S. Succi, The lattice Boltzmann equation: for fluid dynamics and beyond, Oxford university press, 2001.

[5] K. Xu, A gas-kinetic BGK scheme for the NavierCStokes equations and its connection with artificial dissipation and Godunov method, J. Comput. Phys., 171 (1) (2001), 289-335.

[6] K. Xu and J.C. Huang, A unified gas-kinetic scheme for continuum and rarefied flows, J. Comput. Phys., 229 (2010), 7747-7764.

[7] Z.L. Guo, K. Xu, and R.J. Wang, Discrete unified gas kinetic scheme for all Knudsen number flows: Low-speed isothermal case, Phys. Rev. E, 88 (2013), 033305.

[8] R.A. Gingold and J.J. Monaghan, Smoothed particle hydrodynamics-theory and application to non-spherical stars, Mon. Roy. Astron. Soc., 181 (1977), 375-389.

[9] D. Yu, R. Mei, L.-S. Luo, and W. Shyy, Viscous flow computations with the method of lattice Boltzmann equation, Prog. Aerospace Sci., 39 (2003), 329-367.

[10] K. Xu and S.H. Lui, Rayleigh-Benard simulation using the gas-kinetic BhatnagarCGrossCKrook scheme in the incompressible limit, Phys. Rev. E, 60 (1) (1999), 464-470.

[11] X. Shan and H. Chen, Lattice Boltzmann model for simulating flows with multiple phases and components, Phys. Rev. E, 47 (1993), 1815-1819.

[12] L.-S. Luo, Unified theory of the lattice Boltzmann models for nonideal gases, Phys. Rev. Lett., 81 (1998), 1618-1621.

[13] L.-S. Luo, Theory of lattice Boltzmann method: lattice Boltzmann models for nonideal gases, Phys. Rev. E, 62 (2000), 4982-4996.

[14] Z.L. Guo and T.S. Zhao, Discrete velocity and lattice Boltzmann models for binary mixtures of nonideal fluids, Phys. Rev. E, 68 (2003), 035302.

[15] L.-S. Luo and S.S. Girimaji, Lattice Boltzmann model for binary mixtures, Phys. Rev. E, 66 (2002), 035301(R). 
[16] L.-S. Luo and S.S. Girimaji, Theory of the lattice Boltzmann method: two-fluid model for binary mixtures, Phys. Rev. E, 67 (2003), 036302.

[17] K. Xu, Super-Burnett solutions for Poiseuille flow, Phys. Fluid, 15 (2003), 2077-2080.

[18] K. Xu and Z.H. Li, Microchannel flow in the slip regime: gas-kinetic BGK-Burnett solutions, J. Fluid Mech., 513 (2004), 87-110.

[19] Y. Qian, D. d'Humires, and P. Lallemand, Lattice BGK models for Navier-Stokes equation, Europhys. Lett., 17 (1992), 479.

[20] H. Chen, S. Chen, and W.H. Matthaeus, Recovery of the Navier-Stokes equations using a lattice-gas Boltzmann method, Phys. Rev. A, 45 (1992), R5339-R5342.

[21] S. Hou, Q. Zou, S. Chen, and G. Doolen, A.C. Cogley, Simulation of cavity flow by the lattice Boltzmann method, J. Comput. Phys., 118 (1995), 329-347.

[22] L.-S. Luo, W. Liao, X. Chen, Y. Peng, and W. Zhang, Numerics of the lattice Boltzmann method: Effects of collision models on the lattice Boltzmann simulations, Phys. Rev. E, 83 (2011), 056710.

[23] P. Lallemand and L.-S. Luo, Theory of the lattice Boltzmann method: Dispersion, dissipation, isotropy, Galilean invariance, and stability, Phys. Rev. E, 61 (2000), 6546.

[24] Z.L. Guo, and C. Shu, Lattice Boltzmann method and its application in engineering, World Scientific press, 2013.

[25] N. Cao, S. Y. Chen, and D. Martinez, Physical symmetry and lattice symmetry in the lattice Boltzmann method, Phys. Rev. E, 55 (1997), 21-24.

[26] Z.L. Guo, and T. S. Zhao, Explicit finite-difference lattice Boltzmann method for curvilinear coordinates, Phys. Rev. E, 67 (2003), 066709

[27] G. Peng, H. Xi, C. Duncan and S. H. Chou, Finite volume scheme for the lattice Boltzmann method on unstructured meshes, Phys. Rev. E, 59 (1999), 4675.

[28] N. Rossi1, S. Ubertini1, G. Bella1, and S. Succi, Unstructured lattice Boltzmann method in three dimensions. Int. J. Numer. Meth. Fluids, 49 (2005), 619-633.

[29] S. Ubertini and S. Succi, Recent advances of lattice Boltzmann techniques on unstructured grids, Prog. Comput. Fluid Dyn., 5(1/2) (2005), 85-96.

[30] S. Ubertini and S. Succi, A generalised lattice Boltzmann equation on unstructured grids, Commun. Comput. Phys., 3 (2008), 342

[31] T. Lee, and C.-L. Lin, A characteristic Galerkin method for discrete Boltzmann equation, J. Comput. Phys., 171 (2001), 336-356.

[32] P.L. Bhatnagar, E.P. Gross, and M. Krook, A model for collision processes in gases. I. Small amplitude processes in charged and neutral one-component systems, Phys. Rev., 94 (1954), 511.

[33] U. Ghia, K.N. Ghia, and C. Shin, High-Re solutions for incompressible flow using the NavierStokes equations and a multigrid method, J. Comput. Phys., 48 (1982), 387-411.

[34] O. Botella and R. Peyret, Benchmark spectral results on the lid-driven cavity flow, Comput. Fluid, 27 (1998), 421-433.

[35] M. Breuer, J. Bernsdorf, T. Zeiser, and F. Durst, Accurate computations of the laminar flow past a square cylinder based on two different methods: lattice-Boltzmann and finite-volume, Int. J. Heat Fluid Flow, 21 (2000), 186-196.

[36] Z.L. Guo, H.W. Liu, L.S. Luo, and K. Xu, A comparative study of the LBE and GKS methods for 2D near incompressible laminar flows, J. Comput. Phys., 227 (2008), 4955-4976.

[37] A. Ladd and R. Verberg, Lattice-Boltzmann simulations of particle-fluid suspensions, J. Stat. Phys., 104 (2001), 1191-1251.

[38] S. Turki, H. Abbassi, and S.B. Nasrallah, Effect of the blockage ratio on the flow in a channel with a built-in square cylinder, Comput. Mech., 33 (2003), 22-29. 